A.T. GEORGES

N.E. KARATZAS

\title{
Theory of multiple harmonic generation in reflection from a metal surface
}

Department of Physics, University of Patras, Patras 26500, Greece

Published online: 23 August 2005 - C Springer-Verlag 2005

Appl. Phys. B

DOI: $10.1007 / \mathrm{s} 00340-005-1880-8$

Published online: 21 June 2005

Unfortunately, Eqs. (8), (9), (10) and (17) as well as the equations in the first line after Eq. (15) and the fourth line after Eq. (19) were printed with errors.

The corrected text including Eqs. (8)-(19) is printed here:

Since the effective thickness of the metallic surface layer that is excited by the laser field is one skin depth, which is much smaller than the wavelength of the generated harmonics in vacuum, we can treat the polarized layer as a polarization sheet at $z=0$. Integrating Eq. (6) over time and using the rotating wave approximation, we find that the quantum operator for the nonlinear surface polarization at the $N$ th harmonic of the laser frequency can be written as

$\hat{P}_{N \mathrm{sz}}(x, t)=\hat{\mathcal{P}}_{N \mathrm{sz}}(t) \mathrm{e}^{\mathrm{i} N\left(\omega t-k_{\mathrm{x}} x\right)}+$ h.c.,

with the amplitude being

$\hat{\mathcal{P}}_{N \mathrm{sz}}(t)=\frac{e}{\mathrm{i} N \omega m} \frac{d}{V} \sum_{j}\left[p_{\mathrm{z}, N 0} \hat{\sigma}_{N, 0}(t)-e \mathcal{A}(t) \hat{\sigma}_{N-1,0}(t)\right]_{j}$,

where the sum is over all electrons, $d$ is the skin depth of the metal, $V$ the volume of the excited surface layer, $\hat{\sigma}_{N, 0}=|N\rangle\langle 0| \mathrm{e}^{-\mathrm{i} N\left(\omega t-k_{\mathrm{x}} x\right)}$ a slowly varying electron-raising operator, and $\mathcal{A}(t)$ the slowly varying complex amplitude of the $z$ component of the vector potential. The expectation value of the amplitude of the surface polarization is simply

$$
\left\langle\hat{\mathcal{P}}_{N \mathrm{sz}}(t)\right\rangle=\frac{e}{\mathrm{i} N \omega m} \frac{d}{V} \sum_{j}\left[p_{\mathrm{z}, N 0} \sigma_{0, N}(t)-e \mathcal{A}(t) \sigma_{0, N-1}(t)\right]_{j},
$$

Fax: +30-2610-997-467, E-mail: georges@ danaos.physics.upatras.gr

The online version of the original article can be found at http://dx.doi.org/10.1007/s00340-005-1880-8 where we have used the relation $\left\langle\hat{\sigma}_{N, 0}\right\rangle=\operatorname{Tr}\left[\rho \hat{\sigma}_{N, 0}\right]=\sigma_{0, N}$, with $\sigma_{0, N}$ being a slowly varying density-matrix element. The two terms on the right-hand side of the equation above correspond to different processes contributing to the nonlinear polarization at $N \omega$. The first term is associated with $N$-photon absorption followed by emission of a photon of frequency $N \omega$. The second term, on the other hand, is associated with inelastic scattering of a laser photon from the $(N-1)$-photon excited electron state to produce a photon of frequency $N \omega$. Note that, for $N=1$, keeping only the second term in Eq. (9) and introducing the Drude damping rate, we recover the classical result for the bulk polarization of a free-electron gas at the fundamental frequency,

$$
\left\langle\hat{\mathcal{P}}_{\omega, \mathrm{z}}\right\rangle=-\frac{e^{2}}{\mathrm{i}\left(\omega-\mathrm{i} \Gamma_{\mathrm{D}}\right) m} \mathcal{A} \frac{1}{V} \sum_{j} \sigma_{00, j}=-\epsilon_{0} \frac{\omega_{\mathrm{p}}^{2}}{\omega\left(\omega-\mathrm{i} \Gamma_{\mathrm{D}}\right)} \mathcal{E},
$$

where $\omega_{\mathrm{p}}=\left(N_{\mathrm{e}} e^{2} / m \epsilon_{0}\right)^{1 / 2}$ is the plasma frequency, with $N_{\mathrm{e}}$ being the bulk electron density.

\subsection{Harmonic radiation fields}

The quantum-mechanical nonlinear surface polarization gives rise to harmonic fields both in reflection and transmission from the metal surface,

$\hat{\mathbf{E}}_{N \mathrm{r}}(x, z, t)=\hat{\mathcal{E}}_{N \mathrm{r}}(t) \exp \left[\mathrm{i}\left(N \omega t-k_{N \mathrm{rx}} x-k_{N \mathrm{rz}} z\right)\right]+$ h.c.,

$\hat{\mathbf{E}}_{N \mathrm{t}}(x, z, t)=\hat{\mathcal{E}}_{N \mathrm{t}}(t) \exp \left[\mathrm{i}\left(N \omega t-k_{N \mathrm{tx}} x+k_{N \mathrm{tz}} z\right)\right]+$ h.c.,

where $\hat{\mathcal{E}}_{N \mathrm{r}}(t)$ and $\hat{\mathcal{E}}_{N \mathrm{t}}(t)$ are the quantum-mechanical vector amplitudes of the reflected and transmitted electric fields, 
$k_{N \mathrm{rx}}=(N \omega / c) \sin \vartheta_{r}$ and $k_{N \mathrm{rz}}=(N \omega / c) \cos \vartheta_{\mathrm{r}}$ are the wavevector components of the reflected harmonic field with $\vartheta_{\mathrm{r}}$ being the angle of reflection, while $k_{N \mathrm{tx}}$ and $k_{N \mathrm{tz}}=k_{N \mathrm{tz}}^{\prime}$ $\mathrm{i} k_{N \mathrm{tz}}^{\prime \prime}$ are the wave-vector components of the transmitted field. Applying boundary conditions for the tangential components of the electric and magnetic fields in the case of a $z$-polarized dipole sheet $[4,5,12]$,

$\hat{E}_{N \mathrm{rx}}-\hat{E}_{N \mathrm{tx}}=\frac{1}{\epsilon(N \omega)} \frac{\partial \hat{P}_{N \mathrm{sz}}}{\partial x}$,

and

$\mathbf{k}_{N \mathrm{r}} \times \hat{\mathbf{E}}_{N \mathrm{r}}=\mathbf{k}_{N \mathrm{t}} \times \hat{\mathbf{E}}_{N \mathrm{t}}$,

one can prove that: (i) $\vartheta_{\mathrm{r}}=\vartheta_{\mathrm{i}}$ for all harmonic fields, (ii) the reflected harmonic fields are polarized in the same direction as the reflected fundamental field, and (iii) the quantum amplitude of the electric field of the $N$ th harmonic generated in reflection is given by

$\hat{\mathcal{E}}_{N \mathrm{r}}(t)=\mathrm{i} \frac{N \omega F}{c \epsilon_{0}} \hat{\mathcal{P}}_{N \mathrm{sz}}(t)$,

where $F=\sin \vartheta_{\mathrm{i}} /\left(n_{N \omega}^{2} \cos \vartheta_{\mathrm{i}}+n_{N \omega} \cos \vartheta_{N \mathrm{t}}\right)$ is a Fresnel coefficient for the $N$ th harmonic, with $n_{N \omega}$ being the index of refraction of the metal at $N \omega$ and $\vartheta_{N \mathrm{t}}$ the angle of refraction for the transmitted $N$ th harmonic. It should be noted here that the simple analytical relation between the quantum amplitudes for the radiated harmonic field and the nonlinear polarization is made possible because of the justified approximation to treat the polarized surface layer as a sheet. This eliminates propagation effects and simplifies the theoretical description. From the quantum-mechanical amplitude of the harmonic fields we can calculate not only the intensity of the coherent harmonic radiation, but also the average intensity of the incoherent harmonic component.

The intensity of the coherent component of the reflected $N$ th harmonic, which is associated with the expectation value of the surface polarization, is given by

$I_{N \omega, \operatorname{coh}}(t)=2 c \epsilon_{0} \frac{(N \omega)^{2}|F|^{2}}{\left(c \epsilon_{0}\right)^{2}}\left|\left\langle\hat{\mathcal{P}}_{N \mathrm{sz}}(t)\right\rangle\right|^{2}$,

with

$$
\begin{aligned}
& \left\langle\hat{\mathcal{P}}_{N \mathrm{sz}}\right\rangle=\frac{e}{\mathrm{i} N \omega m} \frac{d}{4 \pi^{3}} \\
& \quad \times \iint\left[p_{\mathrm{z}, N 0} \sigma_{0, N}-e \mathcal{A} \sigma_{0, N-1}\right]_{\kappa_{\mathrm{i}}, \Omega_{\mathrm{i}}} P_{\mathrm{FD}}\left(E_{\mathrm{i}}\right)\left[1-P_{\mathrm{FD}}\left(E_{\mathrm{i}}\right)\right] \\
& \quad \times\left[1-P_{\mathrm{FD}}\left(E_{\mathrm{i}}+\hbar \omega\right)\right] \cdots \\
& \quad \times\left[1-P_{\mathrm{FD}}\left(E_{\mathrm{i}}+N \hbar \omega\right)\right] \kappa_{\mathrm{i}}^{2} \mathrm{~d} \kappa_{\mathrm{i}} \mathrm{d} \Omega_{\kappa_{\mathrm{i}}},
\end{aligned}
$$

where for computational purposes the sum over electrons in Eq. (9) has been replaced by integration over the Fermi sphere, with $E_{\mathrm{i}}, \kappa_{\mathrm{i}}$, and $\Omega_{\mathrm{i}}$ being the initial electron energy, wave number, and solid angle, respectively. $P_{\mathrm{FD}}\left(E_{\mathrm{i}}\right)$ is the Fermi-Dirac probability distribution, while $\left[1-P_{\mathrm{FD}}\left(E_{\mathrm{i}}+n \hbar \omega\right)\right], \quad n=$ $0,1,2, \ldots$, is the probability that the energy level at $E_{\mathrm{i}}+n \hbar \omega$ is not occupied.

Since for a typical laser intensity of $10 \mathrm{GW} / \mathrm{cm}^{2}$ incident on a metal surface a large percentage of conduction electrons with initial energy near the Fermi energy are in excited states, we need to consider their contribution to incoherent harmonic radiation. The incoherent component of the $N$ th harmonic radiation field arises from the quantum fluctuations in the polarization, $\delta \hat{\mathcal{P}}_{N \mathrm{sz}}=\hat{\mathcal{P}}_{N \mathrm{sz}}-\left\langle\hat{\mathcal{P}}_{N \mathrm{sz}}\right\rangle$, and they become large when there is a large excited population [20]. Using the relation $\left\langle\delta \hat{\mathcal{P}}_{N \mathrm{sz}} \delta \hat{\mathcal{P}}_{N \mathrm{sz}}^{\dagger}\right\rangle=\left\langle\hat{\mathcal{P}}_{N \mathrm{sz}} \hat{\mathcal{P}}_{N \mathrm{Nz}}^{\dagger}\right\rangle-\left|\left\langle\hat{\mathcal{P}}_{N \mathrm{sz}}\right\rangle\right|^{2}$, the average intensity of the incoherent component of the reflected $N$ th harmonic can be written as

$I_{N \omega, \text { inc }}(t)=2 c \epsilon_{0} \frac{(N \omega)^{2}|F|^{2}}{\left(c \epsilon_{0}\right)^{2}}\left\langle\delta \hat{\mathcal{P}}_{N \mathrm{sz}} \delta \hat{\mathcal{P}}_{N \mathrm{sz}}^{\dagger}\right\rangle$,

where

$$
\begin{aligned}
\left\langle\delta \hat{\mathcal{P}}_{N \mathrm{sz}} \delta \hat{\mathcal{P}}_{N \mathrm{sz}}^{\dagger}\right\rangle= & \frac{e^{2}}{(m N \omega)^{2}} \frac{d^{2}}{V^{2}} \sum_{j}\left\langle\left[ p_{\mathrm{z}, N 0} \hat{\sigma}_{N, 0}\right.\right. \\
& \left.\left.-e \mathcal{A} \hat{\sigma}_{N-1,0}\right]\left[p_{\mathrm{z}, 0 N} \hat{\sigma}_{0, N}-e \mathcal{A}^{*} \hat{\sigma}_{0, N-1}\right]\right\rangle_{j} \\
= & \frac{e^{2}}{(m N \omega)^{2}} \frac{d}{A_{\mathrm{s}}} \frac{1}{V} \sum_{j}\left\{\left|p_{z, 0 N}\right|^{2} \sigma_{N, N}(t)\right. \\
& +|e \mathcal{A}(t)|^{2} \sigma_{N-1, N-1}(t) \\
& \left.-2 \operatorname{Re}\left[p_{\mathrm{z}, N 0} e \mathcal{A}^{*}(t) \sigma_{N-1, N}(t)\right]\right\}_{j},
\end{aligned}
$$

with $A_{\mathrm{s}}=V / d$ being the excited surface area. To gain more physical insight we use the expression for the classical electron radius, $r_{0}=e^{2} /\left(4 \pi \epsilon_{0} m c^{2}\right)$, and the relation $e p_{\mathrm{z}, 0 N}=\mathrm{i} m N \omega \mu_{\mathrm{z}, 0 N}$ between the matrix elements of the momentum operator and the electric dipole $(\mu=e r)$, to recast the expression for the intensity of the incoherent component in the form

$$
\begin{aligned}
I_{N \omega, \text { inc }}(t)= & \frac{1}{V} \sum_{j} d|F|^{2}\left\{\sigma_{N, N}(t) \hbar N \omega \gamma_{\mathrm{sp}, N}\right. \\
& +\sigma_{N-1, N-1}(t) I_{\omega}(t) r_{0}^{2} \Delta \Omega_{1} \\
& \left.-2 \operatorname{Re}\left[\sigma_{N-1, N}(t)\right]\left[\hbar N \omega \gamma_{\mathrm{sp}, N} I_{\omega}(t) r_{0}^{2} \Delta \Omega_{1}\right]^{1 / 2}\right\}_{j},
\end{aligned}
$$

where

$\gamma_{\mathrm{sp}, N}=\frac{(N \omega)^{3}\left|\mu_{N 0, \mathrm{i}}\right|^{2}}{8 \pi^{2} \epsilon_{0} c^{3} \hbar} \Delta \Omega_{N}$

is the spontaneous emission rate from the $N$-photon excited state to the ground state within the solid angle $\Delta \Omega_{N}=\left(2 \lambda_{N}\right)^{2} / A_{\mathrm{s}}$, with $\lambda_{N}$ being the wavelength of the $N$ th harmonic. 